\title{
Dynamics of holographic thermalization
}

\author{
Walter Baron ${ }^{a, 1}$, Damián Galante $e^{b, c, 2}$ and Martin Schvellinger ${ }^{a, 3}$ \\ ${ }^{a}$ IFLP-CCT-La Plata, CONICET and Departamento de Física, Universidad Nacional de \\ La Plata. Calle 49 y 115, C.C. 67, (1900) La Plata, Buenos Aires, Argentina. \\ ${ }^{b}$ Perimeter Institute for Theoretical Physics, Waterloo, Ontario N2J 2W9, Canada. \\ ${ }^{c}$ Department of Applied Mathematics, University of Western Ontario, \\ London, Ontario N6A 5B\%, Canada.
}

\begin{abstract}
Dynamical evolution of thin shells composed by different kinds of degrees of freedom collapsing within asymptotically AdS spaces is explored with the aim of investigating models of holographic thermalization of strongly coupled systems. From the quantum field theory point of view this corresponds to considering different thermal quenches. We carry out a general study of the thermalization time scale using different parameters and space-time dimensions, by calculating renormalized space-like geodesic lengths and rectangular minimal area surfaces as extended probes of thermalization, which are dual to two-point functions and rectangular Wilson loops. Different kinds of degrees of freedom in the shell are described by their corresponding equations of state. We consider a scalar field, as well as relativistic matter, a pressureless massive fluid and conformal matter, which can be compared with the collapse of an AdS-Vaidya thin shell. Remarkably, in the case of $\mathrm{AdS}_{5}$, for conformal matter, the thermalization time scale becomes much larger than the others. Furthermore, in each case we also investigate models where the cosmological constants of the inner and outer regions separated by the shell are different. We found that in this case only a scalar field shell collapses, and that the thermalization time scale is also much larger than the AdS-Vaidya case.
\end{abstract}

\footnotetext{
${ }^{1}$ wbaron@fisica.unlp.edu.ar

${ }^{2}$ dgalante@perimeterinstitute.ca

${ }^{3}$ martin@fisica.unlp.edu.ar
} 


\section{Contents}

1 Introduction and motivation $\quad 2$

2 Thermal quenches and equations of state 5

2.1 Shell velocity . . . . . . . . . . . . . . . . . . 5

2.2 Mass function . . . . . . . . . . . . . . . . . . . . 8

2.3 Dynamical evolution of shells of matter . . . . . . . . . . . . . 9

3 Holographic thermalization $\quad 10$

3.1 Renormalized geodesics lengths . . . . . . . . . . . . . . . . 12

3.2 Renormalized rectangular minimal area surfaces . . . . . . . . . . . . 13

4 Results of dynamical holographic thermalization $\quad 14$

5 Discussion and conclusions $r$ 


\section{Introduction and motivation}

The idea of the present work is to investigate different kinds of consistent holographic thermal quenches modeling thermalization processes in strongly coupled systems. As we shall explain in detail, by construction, they satisfy the general relativity equations of motion and the positive energy conditions. Our particular interest is focused on the strongly coupled quarkgluon plasma (QGP) produced by the collision of heavy ions at the Relativistic Heavy Ion Collider (RHIC) and the Large Hadron Collider (LHC). As it is well-known the formation and evolution of a quark-gluon plasma can be viewed as a sequence four distinct steps. First, two heavy ions, typically gold nuclei, move towards each other at relativistic velocities, having kinetic energies of order $100 \mathrm{GeV} /$ nucleon. Next, an almond-shape region where the two nuclei collide is developed, and a part of their kinetic energy transforms into intense heat, leading to the beginning of formation of the plasma of quarks and gluons. This is what has been called thermalization of the plasma. When the thermalization is completed the resulting system is a strongly coupled QGP. After a very short while the system expands, cools down and, finally, a multitude of hadrons emerges from the plasma. Given the fact that the QGP in thermal equilibrium behaves as an strongly coupled system, a reasonable working hypothesis is that the thermalization described above may also occur within a strongly coupled regime of QCD. In a number of interesting articles thermalization has been addressed using the gauge/gravity duality $[1,2,3]$. In these papers the dual process is modeled as the collapse of a thin shell moving at the speed of light, using an AdS-Vaidya type metric, which represents a thermal quench $[4,5,6,7,8,9,10,11,12,13,14,15,16,17,18,19]$. Interestingly, there have also been studies on holographic thermalization described as a dual process of black hole formation [20, 21, 22, 23, 24, 25, 26, 27]. In addition, to our knowledge, reference [28] has been the first one to consider a gravity dual picture of the entire process of strongly coupled supersymmetric Yang-Mills (SYM) plasma formation and cooling using a model where the scattering process initially creates a holographic shower in the AdS bulk. It has been argued [28] that the subsequent gravitational fall leads to a moving black hole, which is the gravity dual model corresponding to an expanding and cooling heavy-ion fireball. Moreover, very recently, it has been investigated the high and low temperature behavior of non-local observables in strongly coupled gauge theories that are dual to AdS space-time [29].

In the present case we shall consider a holographic dual description of the thermalization process. From the point of view of the boundary quantum field theory (QFT), the initial state that one considers is a system at zero temperature. Then, there is a sudden injection of energy which induces an abrupt change in the state of the system. The system evolves leading to a final thermal state which will be a strongly coupled SYM plasma. A very important question is how to model the thermal evolution of the system from the zero temperature state towards the thermally equilibrated SYM plasma, keeping in mind that the initial condition 
is a thermal quench instead of an adiabatic change. Indeed, this is a very hard problem if one tries to study its dynamics in terms of QFT methods. On the other hand, the holographic evolution of a thermal quench can be easily followed by numerical calculations in its gravity dual model. So far, the studies [4]-[19] have considered the evolution of an AdS-Vaidya thin shell, even though at the moment it is not known how to get the initial Vaidya shell condition from a QFT evolution. On the gravity side, we know that it is also possible to solve the equations of motion (EOM) of shells composed by different degrees of freedom, i.e. whose dynamics is described by different equations of state (EOS). Depending on the particular EOS the shells will move and collapse at different velocities in the bulk. This is very interesting since it allows us to investigate the thermalization time scale of different kind of shells. On the boundary theory side, after the collision occurs, the system evolves in a certain way until it reaches thermal equilibrium. On the other hand, on the holographic gravitational dual model, this should be reflected on the evolution of a collapsing shell, which depends on the EOS governing the degrees of freedom which compose it. Thus, we shall be focused at investigating the variation of the thermalization time scale of two-point functions of gauge invariant local operators and rectangular Wilson loops, by calculating their dual renormalized space-like geodesic lengths and rectangular minimal area surfaces. These are extended probes within the dual geometry for thermal equilibrium in the dual QFT. The interesting new feature of this work is that we change the nature of the shell composition. We study different kinds of degrees of freedom in the shell which are described by distinct EOS. These include a scalar field, conformal matter, relativistic matter and a pressureless massive fluid, which can be compared with the collapse of an AdS-Vaidya thin shell. Furthermore, we also investigate models where the cosmological constants of the inner and outer regions are different. On the field theory side this corresponds to changes in the coupling of a SYM theory at zero temperature compared with the SYM plasma coupling at thermal equilibrium.

It is worth noting that for a SYM plasma in thermal equilibrium the system is probed at momentum scales below the equilibrium temperature $T$. This is the so-called hydrodynamical regime, at which the gauge/string duality has been proved to be particularly useful. An important number of investigations have been done in this framework. A very important work in this context is the one of reference [30], where it has been calculated the shear viscosity of the finite-temperature $\mathcal{N}=4 S U(N)$ SYM theory plasma, in the large $N$ limit, at the strong-coupling regime. The first leading order string theory corrections to the shear viscosity over entropy density ratio of strongly coupled SYM plasmas has been obtained in [31]. Besides, electrical charge transport coefficients of strongly coupled SYM plasmas have also been investigated within the gauge/string duality. These include the electrical conductivity, which in the large coupling limit was firstly calculated in [32], while finite 't Hooft coupling corrections were obtained from type IIB string theory corrections at order $\alpha^{\prime 3}$ in [33, 34]. Additionally, the photoemission rates of this plasma have been computed in [35] using the gauge/string duality, while the corresponding leading order string theory 
corrections have been reported more recently in $[36,37]$. Besides, holographic photon and dilepton production in a thermalizing plasma have been investigated within the quasi-static approximation [38, 39, 40].

We would like to emphasize some interesting conclusions which follow from our numerical results. We observe that from the curves of thermalization discussed in this paper, by fixing to one both the inner and outer radii, the shells composed by a pressureless massive fluid and by a scalar field are very close to each other, and almost overlap completely the curve corresponding to the AdS-Vaidya shell moving at the speed of light. On the other hand, relativistic matter thermalizes later, depending on its EOS the difference becomes more important, and finally the shell composed by conformal matter thermalizes much later than the time the AdS-Vaidya shell takes to collapse. We would like to emphasize that this large thermalization time delay is a very remarkable effect, since we think that it opens the possibility of developing new type of models showing slower thermalization in comparison with the AdS-Vaidya models. This is for both space-like renormalized geodesic lengths for space-time dimensions $d=2,3$ and 4, and for renormalized rectangular minimal area surfaces for $d=3$ and 4 . We also have numerically investigated what happens when both radii are equal to each other but we change both simultaneously. Then, we study the effect on the thermalization curves when the inner and outer radii are different. We have obtained an interesting analytical result, namely: the positive energy condition implies that the inner radius must be equal or smaller than the outer one, i.e. the absolute value of the vacuum energy density of the inner region must be equal or larger than the one of the outer region. In addition, only in the case of a shell composed by a scalar field the positive energy condition allows for the collapse of the shell separating regions with different inner and outer vacuum energy density to be produced.

This paper is organized as follows. In section 2 we introduce the formalism, including the description of the thermal quenches corresponding to different kinds of degrees of freedom living in the collapsing shells. We derive the expressions for the velocity of the shell and its mass function. Then, we describe the strongly coupled SYM plasma thermalization process in terms of the evolution of a massive shell. We introduce the extensive gravitational probes we use to measure the thermalization time scale, which includes renormalized space-like geodesic lengths and rectangular minimal area surfaces. The latter correspond to Wilson loops in a 4-dimensional QFT on the boundary, and are proportional to entanglement entropy for a 3-dimensional boundary QFT. These are introduced in section 3. In section 4 we present our results on holographic thermalization for different dimensions of space-time and by exploring different sets of parameters. In the last section we discuss the results. 


\section{Thermal quenches and equations of state}

The dynamics of a massive thin shell is determined by the Israel junction conditions [41]. The shell separates two different geometries, each one being a solution of the Einstein equations, and the Israel's conditions tell us how to match them.

Since the shell is massive, the inner solution will typically be a vacuum one, while the outer geometry will be described by an AdS-Schwarzschild-type solution. The shell can be made of ordinary particles, like null dust as described by the AdS-Vaidya solution, which gives for instance the geometry generated by a spherically symmetric beam of photons in the Eikonal approximation [42, 43]; by conformal matter as described in [44]; moreover, it can also be interpreted as the domain wall of a solitonic solution connecting the inner and outer geometries with different cosmological constants associated with the vacuum expectation value of certain scalar field.

In the next section we will obtain the expression for the velocity of the shell collapsing in a $\operatorname{AdS}_{d+1}$ space-time. The inner geometry will be a pure AdS space, while the outer space will be an asymptotically AdS-Schwarzschild black hole. Notice though that we allow for the radii of both anti-de Sitter spaces to be in principle arbitrary. Moreover, in general terms its evolution can be followed for any EOS governing the degrees of freedom of the shell. Thus, by setting a particular EOS one can determine the velocity of the shell.

\subsection{Shell velocity}

We find useful to describe the AdS spaces by using Eddington-Finkelstein-like coordinates ${ }^{4}$. In terms of these the metrics inside and outside the shell are given respectively by

$$
\begin{aligned}
d s_{\text {in }}^{2} & =g_{M N}^{(\text {in })} d X_{0}^{M} d X_{0}^{N}=\frac{1}{z^{2}}\left(-d v^{2}-2 R_{0} d v d z+d \vec{x}^{2}\right) \\
d s_{\text {out }}^{2} & =g_{M N}^{\text {out })} d X_{f}^{M} d X_{f}^{N}=\frac{1}{z^{2}}\left(-f_{\text {out }}(z) d v^{2}-2 R_{f} d v d z+d \vec{x}^{2}\right)
\end{aligned}
$$

where indices $M$ and $N=1, \cdots, d+1$, while $\vec{x}=\left\{x^{i}\right\}$ with $i=1, \cdots, d-1$ and $R_{0}$ and $R_{f}$ are the AdS radii corresponding to the inner and outer regions, respectively, and

$$
f_{\text {out }}(z)=1-2 M\left(R_{f} z\right)^{d} .
$$

Given the above metrics inside and outside the shell, it is natural to use the following shell embedding metric

$$
d s_{\text {shell }}^{2}=h_{\mu \nu}^{(\text {shell })} d x^{\mu} d x^{\nu}=\frac{1}{z^{2}}\left(-d \tau^{2}+d \vec{x}^{2}\right)
$$

\footnotetext{
${ }^{4}$ The time coordinate is defined as usual through $d t=d v+R_{f} f_{\text {out }}^{-1}(z) d z$.
} 
where we have defined $x^{0} \equiv \tau$, so that $h_{\mu \nu}$ is conformally flat. The proper area of the shell allows us to identify the $z$ variable inside, over and outside the shell.

The energy-momentum tensor necessarily has the form,

$$
T_{M N}=\delta(\eta) S_{M N}-\rho_{0} g_{M N}^{(i n)} \Theta(-\eta)-\rho_{f} g_{M N}^{(\text {out })} \Theta(\eta)
$$

where $\eta$ is the coordinate orthogonal to the shell in the Gaussian normal coordinate system. $S_{M N}$ represents the energy-momentum tensor of the shell. $\rho_{0}$ and $\rho_{f}$ denote the vacuum energy density of the anti-de Sitter spaces, $\rho_{0, f}=\frac{-d(d-1)}{2 \kappa R_{0, f}^{2}}$, with $\kappa=8 \pi G, G$ being the $(d+1)$-dimensional Newton constant.

By computing the divergence of the energy-momentum tensor, $T^{M N}{ }_{; N}$, and demanding the coefficients of $\delta(\eta)$ and $\delta^{\prime}(\eta)$ to vanish separately, it can be shown that the surface energy-momentum tensor must vanish in the normal directions, $S^{M \eta}=0$, and the nontrivial components are conserved in the lower-dimensional sense, i.e.

$$
S^{\mu \nu}{ }_{\mid \nu}=0
$$

where "|" denotes the covariant derivative constructed with $h_{\mu \nu}^{(s h e l l)}$. Another consequence of $T_{; N}^{M N}=0$ is the junction condition

$$
\left\{K_{\mu \nu}\right\} S^{\mu \nu}=\rho_{0}-\rho_{f}
$$

where $\left\{K_{\mu \nu}\right\}=\frac{1}{2}\left[K_{\mu \nu}(\right.$ in $)+K_{\mu \nu}($ out $\left.)\right]$, while $K_{\mu \nu}=n_{\mu ; \nu}$ denotes the extrinsic curvature, $n$ being the normal vector to the shell.

We will consider a shell composed by a perfect fluid, so that

$$
S^{\mu \nu}=z(\tau)^{2}(\epsilon+p) u^{\mu} u^{\nu}+p h^{\mu \nu},
$$

where the velocities $u^{\mu}$ are defined as $\frac{d x^{\mu}}{d \tau}$, with $\tau$ being the conformal time, not to be confused with the proper time. Then, equation (5) implies

$$
\dot{\epsilon}=(d-1) \frac{\dot{z}}{z}(\epsilon+p)
$$

where dot stands for derivative with respect to $\tau$. In the above expression $\epsilon$ is the energy density and $p$ is the pressure within the shell.

Einstein equations of the $(d+1)$-dimensional space-time lead to the so-called Israel junction conditions, namely:

$$
\left[K_{\mu \nu}-h_{\mu \nu}^{(s h e l l)} \operatorname{tr} K\right]=\kappa S_{\mu \nu}
$$

the square bracket $[\cdot]$ denotes the difference of the quantity inside and outside the shell. 
The velocity of the fluid is set to be in the radial direction, so that $u^{\mu} \rightarrow(\dot{v}, \dot{z}, \overrightarrow{0})$, and the normal vector $n^{\mu}$ defined as the unit vector orthogonal to $u^{\mu}$ is easily found and leads to the following extrinsic curvature

$$
\begin{aligned}
K_{x^{i} x^{j}}(\text { out }) & =\frac{\sqrt{f_{\text {out }}+R_{f}^{2} \dot{z}^{2}}}{R_{f} z^{2}} \delta_{i j}, \quad i, j=1,2, \cdots, d-1, \\
K_{\tau \tau}(\text { out }) & =\frac{d}{d z} \frac{\sqrt{f_{\text {out }}+R_{f}^{2} \dot{z}^{2}}}{R_{f} z},
\end{aligned}
$$

in the outer region. In the inner region there are similar expressions by just replacing $f_{\text {out }} \Rightarrow 1$ and $R_{f} \Rightarrow R_{0}$. Then, the Israel junction conditions become

$$
\sqrt{R_{0}^{-2}+\dot{z}^{2}}-\sqrt{f_{\text {out }} R_{f}^{-2}+\dot{z}^{2}}=\frac{\kappa}{d-1} \epsilon .
$$

After some algebra equation (11) leads to

$$
\sqrt{R_{0}^{-2}+\dot{z}^{2}}+\sqrt{f_{\text {out }} R_{f}^{-2}+\dot{z}^{2}}=\frac{d-1}{\kappa \epsilon}\left(R_{0}^{-2}-f_{\text {out }} R_{f}^{-2}\right),
$$

which represents the junction condition (6). In fact, it implies

$$
\epsilon \frac{d\left(z\left\{K_{x^{1} x^{1}}\right\}\right)}{d z}+(d-1) p\left\{K_{x^{1} x^{1}}\right\}=-\frac{d(d-1)}{2 \kappa}\left(\frac{1}{R_{0}^{2}}-\frac{1}{R_{f}^{2}}\right) .
$$

Then, using equation (8) this differential equation can be integrated to obtain equation (12).

Equations (11) and (12) can be used to derive the following expression

$$
\dot{z}^{2}=\frac{h^{2}-2\left(R_{0}^{-2}+f_{\text {out }} R_{f}^{-2}\right) h+\left(R_{0}^{-2}-f_{\text {out }} R_{f}^{-2}\right)^{2}}{4 h},
$$

where in order to make the notation simpler we have introduced $h=\left(\frac{\kappa \epsilon}{d-1}\right)^{2}$.

Since we are assuming that the shell is composed by a perfect fluid the entropy must be a constant, which can be negligibly small such that its EOS can be reduced to $p=p(\epsilon)$. In many physical situations the EOS can exactly or at least approximately be recast in the form $p=a \epsilon$, with $a$ being a constant. For instance, when $a=\frac{1}{d-1}$ it represents a fluid composed by conformal matter (i.e. its degrees of freedom have a traceless energy-momentum tensor). On the other hand, the case with $a=0$ corresponds to dust, while $a=-1$ (see [45]) can be modeled by a scalar field ${ }^{5}$. In cosmological applications these are commonly employed in

\footnotetext{
${ }^{5}$ To see this, notice that the energy-momentum tensor for a scalar field is
}

$$
T_{\mu \nu}=\partial_{\mu} \phi \partial_{\nu} \phi-g_{\mu \nu}\left[\frac{1}{2} \partial^{\alpha} \phi \partial_{\alpha} \phi+V(\phi)\right]
$$


order to describe the radiation, matter and dark energy dominated eras. We will consider all these situations along this paper and, in addition, we will include the case of relativistic matter. With the purpose of illustrating this situation we will take a particular example where $a=\frac{9}{10} \frac{1}{d-1}$ for relativistic matter.

Using this equation of state, equation (8) leads to the energy density

$$
\epsilon=\epsilon_{0} z(\tau)^{A},
$$

where $A=(d-1)(a+1)$ and $\epsilon_{0}$ is set by the initial conditions. For instance, we will fix it by demanding that the shell is at rest at a given position $z=z_{0} \cdot{ }^{6}$ Therefore, one may write

$$
h(z)=\left(R_{0}^{-1}-\sqrt{f_{\text {out }}\left(z_{0}\right)} R_{f}^{-1}\right)^{2}\left(\frac{z}{z_{0}}\right)^{2 A} .
$$

Notice that since the cut-off $z_{0}$ can be arbitrarily small, the weak energy condition requires

$$
R_{f} \geq R_{0} .
$$

Next, we derive the mass function of shells.

\subsection{Mass function}

Strictly speaking the analysis above corresponds to a shell of zero thickness. Nevertheless, for computational purposes we will consider the limiting case of small but non-vanishing width, and we will model the situation with the following metric

$$
d s^{2}=\frac{1}{z^{2}}\left(-f d v^{2}-2 R d v d z+d \vec{x}^{2}\right)
$$

where

$$
\begin{aligned}
f & =1-2 m(v, z)(R z)^{d} \\
R & =R_{0}-\left(R_{0}-R_{f}\right) \frac{m(v, z)}{M} \\
m & =\frac{M}{2}\left[1+\tanh \frac{w(v, z)}{w_{0}}\right]
\end{aligned}
$$

so, using that a the embedding metric of a hypersurface with normal vector $n_{\mu}$ is given by $h_{\mu \nu}^{\text {shell }}=g_{\mu \nu}-n_{\mu} n_{\nu}$ and the fact that in the thin-shell approximation $\partial_{\mu} \phi \propto n_{\mu}$ at leading order, $T_{\mu \nu}$ decompose in the sum of two terms, one proportional to $h_{\mu \nu}$ and the other one to $n_{\mu} n_{\nu}$. On the other hand if we require the shell to be composed by a perfect fluid, the energy momentum tensor must decomposes in a factor proportional to $h_{\mu \nu}$ and a factor proportional to $U_{\mu} U_{\nu}$, with $U_{\mu} \perp n_{\mu}$ being the four-velocity of the fluid. Then the energy momentum tensor is forced to be proportional to the metric, which means $p=-\epsilon$.

${ }^{6}$ Of course, the situation is different for a massless dust fluid. In this case the shell moves at the speed of light, and obviously it cannot be set at rest at any position. Nevertheless, it can be considered as a limiting case with $\dot{z} \rightarrow \infty$. 
being $w_{0}$ the parameter representing the thickness of the shell, while $w(v, z)=0$ is the equation defining the position of the shell in the $(v, z)$-plane. It is useful to define the quantities

$$
\begin{aligned}
& \bar{f}=f(z, w=0)=1-M(\bar{R} z)^{d} \\
& \bar{R}=R(z, w=0)=\frac{R_{0}+R_{f}}{2}
\end{aligned}
$$

By comparing the induced metric (3) with equation (18) one finds $d \tau^{2}=\bar{f} d v^{2}+2 \bar{R} d v d z$. Then, the position $\{v(\tau), z(\tau)\}$ of the shell satisfies the equation

$$
\bar{f} d v=\left(\frac{\sqrt{\bar{f}+\bar{R}^{2} \dot{z}^{2}}}{\dot{z}}-\bar{R}\right) d z
$$

from which we find the equation describing the dynamics of the shell to be $w=0$, with

$$
w=v-\bar{R} \int_{z_{0}}^{z} d z \bar{f}^{-1}\left(\sqrt{\frac{\bar{f} \bar{R}^{-2}+\dot{z}^{2}}{\dot{z}^{2}}}-1\right),
$$

where we set the following initial conditions $z\left(\tau_{0}\right)=z_{0}$ and $v\left(\tau_{0}\right)=0$.

\subsection{Dynamical evolution of shells of matter}

Once the shell is at rest at $z=z_{0}$, in principle it is not guaranteed that it will always collapse. For instance, we may think of the shell as composed not by ordinary matter like baryons or photons, but instead by the energy of a domain wall of a bubble which encloses an AdS space in the interior with a given cosmological constant, and an another AdS space in the outer region, with a different one. Thus, it may occur that depending on the values of the cosmological constants of the inner and outer regions the bubble may collapse or expand.

Therefore, the dynamics of the shell depends of the sign of $\frac{d \dot{z}^{2}}{d z}$ at $z_{0}$. It can be computed from equation (14) and the result is

$$
\left.\frac{d \dot{z}^{2}}{d z}\right|_{z_{0}}=\lambda\left(z_{0}\right) \xi\left(z_{0}\right)
$$

where

$$
\lambda\left(z_{0}\right)=\frac{h\left(z_{0}\right)+R_{0}^{-2}-f_{\text {out }}\left(z_{0}\right) R_{f}^{-2}}{4 h\left(z_{0}\right)}>0 .
$$

The positivity follows from the positive energy condition. On the other hand, $\xi\left(z_{0}\right)$ is defined as

$$
\xi\left(z_{0}\right)=-\left(R_{0}^{-2}-R_{f}^{-2}\right) \frac{A}{z_{0}}+A\left[R_{0}^{-1}-\sqrt{f_{\text {out }}\left(z_{0}\right)} R_{f}^{-1}\right]^{2} z_{0}^{A-1}+(d-A) R_{f} z_{0}^{d-1} .
$$


Notice that in the cases of interest $0 \leq A \leq d$. Hence, the first term in the equation above is negative while the second and third ones are positive. The conclusion is that in the case of equal radii $\xi\left(z_{0}\right)>0$ and, therefore, the shell always collapses.

On the other hand, when $A=0$ (corresponding to $a=-1) \xi\left(z_{0}\right)$ is again positive and so the shell collapses independently of the values $R_{0}$ and $R_{f}$.

The situation changes dramatically when we consider $A \neq 0$ because in the $z_{0} \rightarrow 0$ limit the leading term is the first one which is negative, implying that the shell generically expands.

For a given cut-off $z_{0}$ it can always be possible to find certain radii $R_{f}>R_{0}$ such that $\xi\left(z_{0}\right)>0$. In order to observe collapse the first term must be smaller than the others, therefore $R_{0}$ and $R_{f}$ must be as close as possible. So, let us define $r=R_{f}-R_{0}<<1$ and for simplicity consider $R_{0}=1$. Therefore we find $r<<z_{0} 10^{-2(2 d-1)}$ for conformal matter, $r<<z_{0} 10^{-(2 d-1)}$ for massive dust, and $r<<z_{0} 10^{-2 d}$ for relativistic matter. In order to give an idea of the orders of magnitude involved, for instance in the case of conformal matter in $A d S_{5}$, by setting the initial position of the shell at $z_{0}=10^{-2}$, we need that $R_{f}-R_{0}<<10^{-16}$ for the shell to collapse and, the limiting case $z_{0} \rightarrow 0$ only allows the fluid with $a=-1$, i.e. a scalar field case, to collapse.

The same conclusion holds even if we relax the initial condition $\left.\dot{z}\right|_{z_{0}}=0$. Indeed let us suppose that $\left.\dot{z}\right|_{z_{0}}>0$, and then extrapolate the shell position backward in time. If we assume $\dot{z} \neq 0$ for all $z<z_{0}$, then as $z \rightarrow 0$ the l.h.s. of (11) vanishes if and only if $R_{0}=R_{f}$, but the r.h.s. vanishes for $A>0(a>-1)$.

Then for a collapsing fluid with $a>-1$ and $R_{0} \neq R_{f}$ the shell can not be extrapolated to $z \rightarrow 0$. Its velocity must vanish at a certain position $\tilde{z}_{0}<z_{0}$ and so, in order for the shell to collapse, $r$ must be smaller than in the case with the shell at rest at $z_{0}$.

\section{Holographic thermalization}

In this section we describe the idea of holographic thermalization. We will follow references [9] and [17] and first consider two-point functions of local gauge invariant QFT operators. For this purpose we look at Wightman functions $[9,8]$ of local gauge invariant QFT operators $\mathcal{O}$ of conformal dimension $\Delta$. We are interested in the equal time correlation functions. The point is to study how these correlators change at different times.

On the other hand, using the gauge/string duality it is possible to compute these correlators when the operators are heavy by using geodesics in AdS spaces. We will compute the two-point functions from a path integral as in references [46, 9]

$$
<\mathcal{O}(t, \mathbf{x}) \mathcal{O}\left(t, \mathbf{x}^{\prime}\right)>=\int \mathcal{D} \mathcal{P} e^{i \Delta L(\mathcal{P})} \approx \sum_{\text {geodesics }} e^{-\Delta \mathcal{L}},
$$

where the path integral includes all possible paths connecting the points at the AdS boundary, 
i.e. $(t, \mathbf{x})$ and $\left(t, \mathbf{x}^{\prime}\right)$. In the above expression $L(\mathcal{P})$ is the proper length corresponding to this path. For space-like trajectories $L(\mathcal{P})$ is imaginary. The idea is to make a saddlepoint approximation for $\Delta \gg 1$. Therefore, only geodesics, i.e. trajectories with extreme lengths will contribute. Notice that in the last term $\mathcal{L}$ indicates actual length of the geodesic between the points at the AdS boundary. In this way, there is a direct relation between the logarithm of the equal-time two-point function and the geodesic length between these two points. It is important to be careful while considering these approximations because the geodesic length diverges due to the AdS boundary contributions. Then, one can define a renormalized distance $\delta \mathcal{L} \equiv \mathcal{L}-2 \ln \left(2 / z_{0}\right)$, in terms of the cut-off $z_{0}$ near the boundary, that suppresses the divergent part coming from pure AdS.

The other type of non-local operators that we will be using are spatial Wilson loops, which are non-local gauge invariant operators in the field theory defined as the integral in a closed path $C$ of the gauge field $A$. Wilson loops provide information about the non-perturbative behavior of gauge theories, however, in general it is difficult to compute them. Using the AdS/CFT correspondence its computation can be done straightforwardly. The expectation value of a Wilson loop is related to the string theory partition function with a world-sheet $\Sigma$ extended on the bulk interior, and ending on the closed contour $C$ on the boundary,

$$
<W(C)>=<\frac{1}{N} \operatorname{Tr}\left(\mathcal{P} e^{\oint_{C} A}\right)>=\int \mathcal{D} \Sigma e^{-\Lambda(\Sigma)} \simeq e^{-\frac{1}{\alpha^{\prime}} \mathcal{A}\left(\Sigma_{0}\right)},
$$

where, in the path integral one has to integrate over all the non-equivalent surfaces whose boundary is $\partial \Sigma=C$, at the AdS boundary. $\Lambda(\Sigma)$ is the string action. The last approximation in equation (27) is obtained in the strong coupling regime by carrying out a saddle-point approximation of the string theory partition function. In this way we can reduce the computation of the expectation value of a Wilson loop to determine the surface of minimal area of the classical world-sheet whose boundary is $C$. This will be a solution to the equations of motion of the bosonic part of the string action [47, 48].

These shell-collapsing models based on the AdS/CFT correspondence allow to understand intuitively how the thermalization process takes place. The outer region is described by a AdS-Schwarzschild black hole, while the inner region is still an AdS space. Now, let us use the geodesic approximation to compute the equal-time two-point functions. If the separation of the boundary points is small enough, then the geodesic cannot reach the shell at $w=0$ and, therefore, the geodesic is seen as a purely AdS-Schwarzschild black hole geodesic, i.e. for short distances in the field theory the system seems to be in thermal equilibrium. If we increase the separation between the insertion of the boundary operators, at some point, the geodesic will cross the shell, and there will be a geodesic refraction which will deviate it in comparison with the thermal one. Thus, we can understand why the thermalization proceeds from short to long distances, i.e. QFT ultraviolet degrees of freedom thermalize first [9].

In the next two subsections we discuss in more detail the construction of the renormalized 
geodesic lengths and the renormalized rectangular minimal area surfaces, which we will used to probe thermalization of strongly coupled systems. These two subsections are a generalization of our previous paper [17] from where we follow the notation.

\subsection{Renormalized geodesics lengths}

In this subsection we focus on the evaluation of space-like geodesic lengths as function of both time and boundary separation length. Thus, we will consider space-like geodesics between points $\left(t, x_{1}\right)=\left(t_{0},-\ell / 2\right)$ and $\left(t^{\prime}, x_{1}^{\prime}\right)=\left(t_{0}, \ell / 2\right)$, where $\ell$ is the separation of the AdS boundary points. The orthogonal coordinates are fixed. For instance, for $d=4$ we have $\left(x_{2}, x_{3}\right)=\left(x_{2}^{\prime}, x_{3}^{\prime}\right)$. Therefore, we use as the geodesic parameter the first coordinate $x_{1}$, that we simply call $x$. The solutions to the geodesic equations are given by the functions $v(x)$ and $z(x)$. Inserting a cut-off $z_{0}$ close to the AdS boundary, the boundary conditions become

$$
z(-\ell / 2)=z_{0}, \quad z(\ell / 2)=z_{0}, \quad v(-\ell / 2)=t_{0}, \quad v(\ell / 2)=t_{0} .
$$

Also, $v(x)$ and $z(x)$ are symmetric under reflection $x \rightarrow-x$. The geodesic length is defined as

$$
\mathcal{L}=\int \sqrt{-d s^{2}}=\int_{-\ell / 2}^{\ell / 2} d x \frac{\sqrt{1-2 R(v, z) z^{\prime}(x) v^{\prime}(x)-f(v, z) v^{\prime}(x)^{2}}}{z(x)}
$$

where the prime indicates derivative with respect to $x$. Functions $v(x)$ and $z(x)$ minimize the geodesic length of equation (29). Since there is an $x$-independent Lagrangian, it implies the

existence of one conserved quantity, which is equivalent to the Hamiltonian of the system. In terms of $f(v, z)$, the conservation equation becomes

$$
1-2 R(v, z) z^{\prime} v^{\prime}-f(z, v) v^{\prime 2}=\left(\frac{z_{*}}{z}\right)^{2},
$$

where the following initial conditions at the tip of the geodesic have been used

$$
z(0)=z_{*}, \quad v(0)=v_{*}, \quad v^{\prime}(0)=z^{\prime}(0)=0 .
$$

We can then solve the EOM for $v(x)$ and $z(x)$, obtaining

$$
\begin{aligned}
0= & 1-v^{\prime}(x)^{2} f(v, z)-2 R(v, z) v^{\prime}(x) z^{\prime}(x)-R(v, z) z(x) v^{\prime \prime}(x) \\
& +\frac{1}{2} z(x) v^{\prime}(x)^{2} \partial_{z} f(v, z)-\frac{1}{2} \partial_{v} R(v, z) z(x) v^{\prime}(x)^{2}, \\
0= & v^{\prime \prime}(x) f(v, z)+R(v, z) z^{\prime \prime}(x)+z^{\prime}(x) v^{\prime}(x) \partial_{z} f(v, z) \\
& +\frac{1}{2} v^{\prime}(x)^{2} \partial_{v} f(v, z)+\partial_{z} R z^{\prime}(x)^{2},
\end{aligned}
$$


so we can just use these equations (and the conservation relation) and replace $f(v, z)$ by the ones of interest to this work. Note that for the different radii case, not only mass derivatives will appear but also radius derivatives.

In order to evaluate the geodesic length as a function of $t_{0}$ and the boundary separation $\ell$ we use the boundary conditions

$$
z(\ell / 2)=z_{0}, \quad v(\ell / 2)=t_{0}
$$

Now, the conservation equation and reflection symmetry lead to the on-shell geodesic length given by the following expression

$$
\mathcal{L}\left(\ell, t_{0}\right)=2 \int_{0}^{\ell / 2} d x \frac{z_{*}}{z(x)^{2}}
$$

Then, we must cancel the divergent part: $\delta \mathcal{L}\left(\ell, t_{0}\right)=\mathcal{L}\left(\ell, t_{0}\right)-2 \ln \left(2 / z_{0}\right)$.

Thus, we can calculate how the thermalization process occurs by considering a collapsing thin shell composed by different kind of degrees of freedom. At this point we can start studying numerically the thermalization process, by solving the EOM for different starting $\left(v_{*}, z_{*}\right)$ values. We set the event horizon of the thermalized geometry to be located at a position such that we have always the same temperature at the final state. The results are discussed in the next section, but before we introduce the formulas of rectangular Wilson loops.

\subsection{Renormalized rectangular minimal area surfaces}

Now we carry out the computation of the minimal area surfaces. Using the AdS metric with a shell, the Nambu-Goto action becomes,

$$
\mathcal{A}_{N G}\left(t_{0}, \ell, R_{W L}\right)=\frac{R_{W L}}{2 \pi} \int_{-\ell / 2}^{\ell / 2} d x \frac{\sqrt{1-f(v, z) v^{\prime 2}-2 R(v, z) z^{\prime} v^{\prime}}}{z^{2}}
$$

for boundary rectangles parametrized by the coordinates $\left(x_{1}, x_{2}\right)$. The rest of the coordinates at the AdS boundary are kept fixed. One assumes the translational invariance along $x_{2}$. Then, we will use $x_{1}$ to parametrize the functions $v\left(x_{1}\right)$ and $z\left(x_{1}\right)$ in the $\mathrm{AdS}_{d+1}$, and we call it $x$. Along the $x_{2}$ direction the rectangular path on the boundary has length $R_{W L}$.

As in the previous case, there is no explicit dependence on $x$ and therefore, there is a conserved quantity corresponding to the Hamiltonian. The tip of the surface is $z_{*}$, with $z^{\prime}(0)=v^{\prime}(0)=0$. Then, the conservation equation becomes

$$
1-2 R(v, z) z^{\prime} v^{\prime}-f(v, z) v^{\prime 2}=\left(\frac{z_{*}}{z}\right)^{4} .
$$


The boundary conditions continue to be the same as in the geodesics case,

$$
z(-\ell / 2)=z_{0}, \quad z(\ell / 2)=z_{0}, \quad v(-\ell / 2)=t_{0}, \quad v(\ell / 2)=t_{0} .
$$

Next, we have to minimize the Nambu-Goto action for this geometry. For our set up, these equations become

$$
\begin{aligned}
0= & 2-2 f v^{\prime 2}-4 R z^{\prime} v^{\prime}-R z v^{\prime \prime}+\frac{1}{2} z v^{\prime 2} \partial_{z} f-\left(z v^{\prime 2} \partial_{v} R+\frac{1}{2} z z^{\prime} v^{\prime} \partial_{z} R\right) \\
0= & 2 f^{2} v^{\prime 2}-f\left(2-4 R v^{\prime} z^{\prime}+\frac{1}{2} z v^{\prime 2} \partial_{z} f\right)-z\left(R^{2} z^{\prime \prime}+R \partial_{z} f v^{\prime} z^{\prime}+\right. \\
& \left.+\frac{1}{2} R v^{\prime 2} \partial_{v} f\right)+z\left(f v^{\prime 2} \partial_{v} R-R z^{\prime 2} \partial_{z} R\right)
\end{aligned}
$$

We can again extract the physical information of time and boundary separation length from the boundary conditions (38) and rewrite the on-shell Nambu-Goto action by making use of the conservation equation, obtaining

$$
\mathcal{A}\left(t_{0}, \ell, R_{W L}\right)=\frac{R_{W L}}{\pi} \int_{0}^{\ell / 2} d x \frac{z_{*}^{2}}{z^{4}} .
$$

Finally, we subtract the divergent part from pure AdS space by defining

$$
\delta \mathcal{A}\left(t_{0}, \ell\right)=\frac{\pi}{R_{W L}}\left(\mathcal{A}\left(t_{0}, \ell, R_{W L}\right)-\frac{1}{z_{0}} \frac{R_{W L}}{\pi}\right) .
$$

Now, we focus on the results obtained by solving the differential equations for both renormalized space-like geodesic lengths and rectangular minimal area surfaces.

\section{Results of dynamical holographic thermalization}

In this section we introduce our results obtained from numerical calculations, by solving the system of differential equations described in the previous section for the evolution of thin shells, using renormalized geodesic lengths and rectangular minimal area surfaces as extended probes of thermalization of QFT strongly coupled systems.

First, in figure 1 we show the results for thermalization of the renormalized space-like geodesic lengths for the boundary separation $\ell=2.6$, by considering $R_{0}=R_{f}=1$ and $2 M=1$, for the boundary QFT theory dimensions $d=4,3$ and 2, indicated as $\mathrm{AdS}_{5}, \mathrm{AdS}_{4}$ and $\mathrm{AdS}_{3}$, respectively. The cases with a shell composed of a scalar field (green curve) and dust (orange curve), both almost coincide with the Vaidya shell (red curve) as it is shown in figures 1.a, 1.c and 1.e. It turns out that the Vaidya shell thermalizes first. Slightly later

it does the shell composed by a scalar field and then, almost at the same time the shell of 
dust. In fact, these three cases depicted in figures 1.a, 1.c and 1.e, almost completely overlap. In the same figure the dark-red curve indicates relativistic matter, which thermalizes later. Notice that for relativistic matter, whose EOS has $a=c /(d-1)$, we have the freedom to set $0<c<1$, being the pressureless and conformal matter the limiting cases. As $c$ increases, so does the thermalization time for relativistic matter, approaching the conformal matter time scale. Figures 1.b, 1.d and 1.f show thermalization when considering conformal matter (blue curve), which occurs at $t_{0}$ much larger than the other cases. The fact that AdS-Vaidya, scalar field and dust shells coincide is a general result which does not depend on boundary separation. This is so because the integrand on the r.h.s. of equation (22) is much smaller than one for any value of $z$. Thus, the equation describing the position of the shell is $v \simeq 0$, as in the AdS-Vaidya case. The larger thermalization time found for the conformal matter case is closely related to the fact that the r.h.s. of equation (22) takes a non-zero asymptotic value for large $z$. This value increases with space-time dimension, making conformal matter in higher dimensions to thermalize later.

Another interesting possibility is to consider $R_{0}=R_{f}$ with different values. In fact, we have considered $R_{0}=R_{f}=0.5$ in figures 2.a and 2.b and $R_{0}=R_{f}=2$ in figures 2.c and 2.d. These cases are for systems going from $\mathrm{AdS}_{5}$ to an $\mathrm{AdS}_{5}$-Schwarzschild black hole. In both cases we can see that AdS-Vaidya (red curve), a massive dust (orange curve) and a scalar field (green curve) thermalize almost simultaneously, relativistic matter does it a bit later (dark-red curve), and much later conformal matter (blue curve). This difference can be better appreciated from the insets of both figures. In all these curves we keep the dimensionless product of the boundary separation length by the plasma equilibrium temperature $\ell T$ fixed, thus by changing $R_{f}$ the horizon changes as $z_{h}=1 / R_{f}$.

We can also make a comparison between figures 1 and 2. For the AdS-Vaidya, massive dust, and a scalar field cases the thermalization time scale is not sensitive to the changes of the radii, in the range considered, i.e. $R_{0}=R_{f}=0.5,1$ and 2 . The more remarkable effect is that for conformal matter where for $R_{0}=R_{f}=0.5,1$ and 2 , the thermalization time decreases notoriously, as can be seen from the figures. In the particular case of relativistic matter considered we observe a small enhancement of the thermalization time as the radii increase, but of course the thermalization of this kind of matter strongly depends on the value $a$.

Another situation that we have investigated is the case when the inner and outer radii are different. As it has been explained before, only a shell composed by a scalar field can thermalize in this case. In order to illustrate the behavior we have considered: $R_{0}=0.5$, while $R_{f}=1$ (red curve), $R_{f}=2$ (blue curve). For these cases the thermalization time is $t_{0} \approx 90$ and 60 , respectively, while $\overline{\delta \mathcal{L}}-\overline{\delta \mathcal{L}_{B H}}=-2,-5$. This is shown in figure 3 . By incrementing the difference between $R_{0}$ and $R_{f}$ it is possible to recover short thermalization times, for instance the case with $R_{0}=0.5, R_{f}=10$ gives $t_{0} \approx 10$ (not displayed here). Notice that the thermalization scales are not monotonous with respect to the difference 


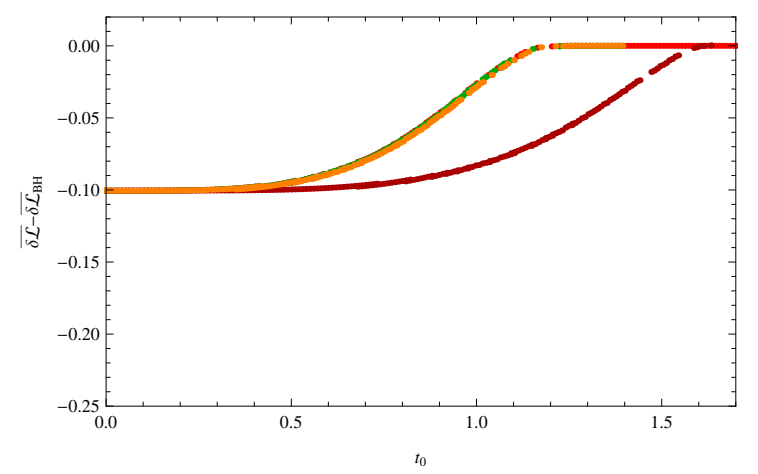

(a) $\mathrm{AdS}_{5}$

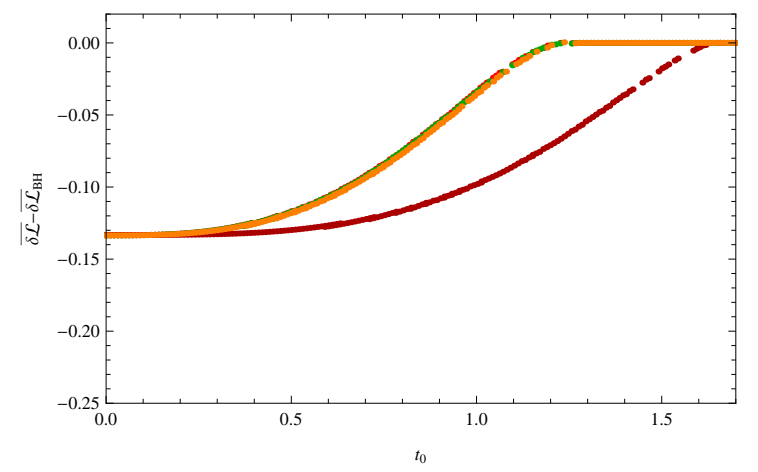

(c) $\mathrm{AdS}_{4}$

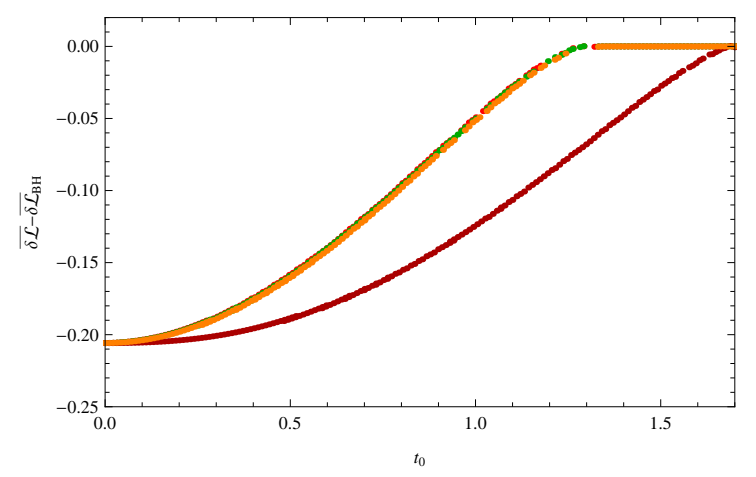

(e) $\mathrm{AdS}_{3}$

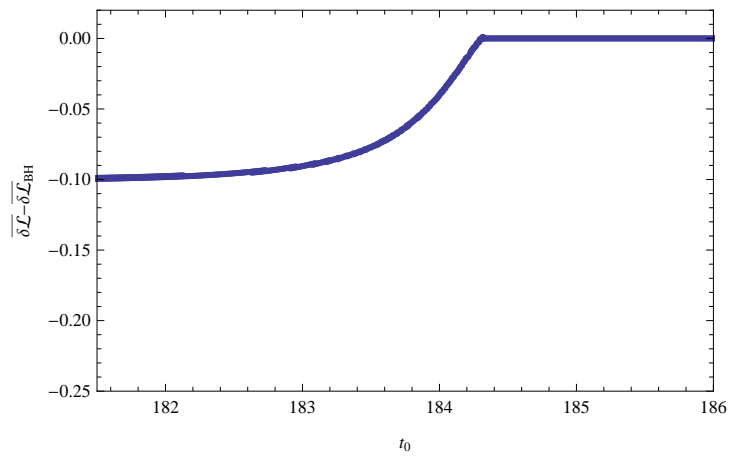

(b) $\mathrm{AdS}_{5}$

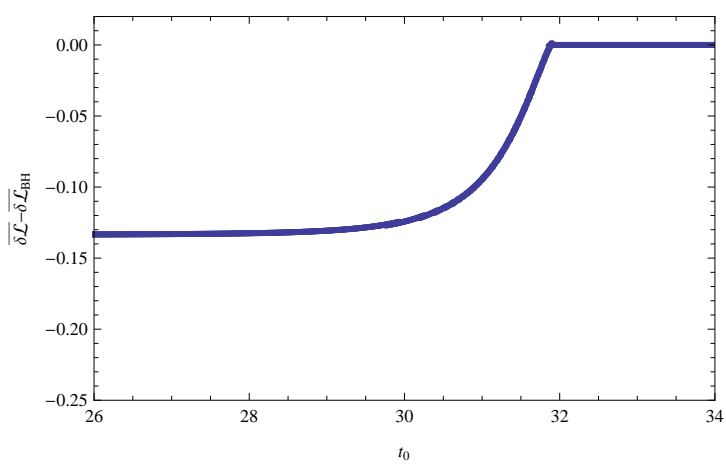

(d) $\mathrm{AdS}_{4}$

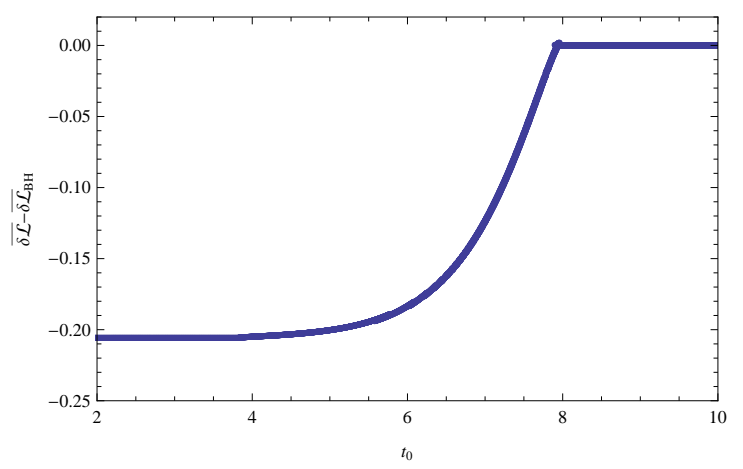

(f) $\mathrm{AdS}_{3}$

Figure 1: Thermalization of the renormalized space-like geodesic lengths for the boundary separation $\ell=2.6$, considering $R_{0}=R_{f}=1$, for boundary theory dimensions $d=4,3$ and 2, indicated as $\mathrm{AdS}_{5}, \mathrm{AdS}_{4}$ and $\mathrm{AdS}_{3}$, respectively. In each figure curves for different matter are indicated with different colors: for AdS-Vaidya (red curve), scalar field (green curve), massive dust (orange curve), relativistic matter (dark red curve), conformal matter (blue curve). Following the literature we plot the difference between the geodesic length and the thermal geodesic length divided by the boundary separation $\ell$. The same applies for the rest of the figures. 


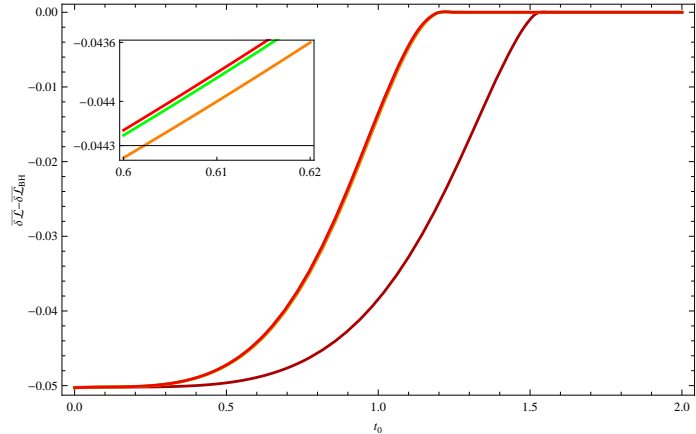

(a) $\mathrm{AdS}_{5}, R_{0}=R_{f}=0.5$

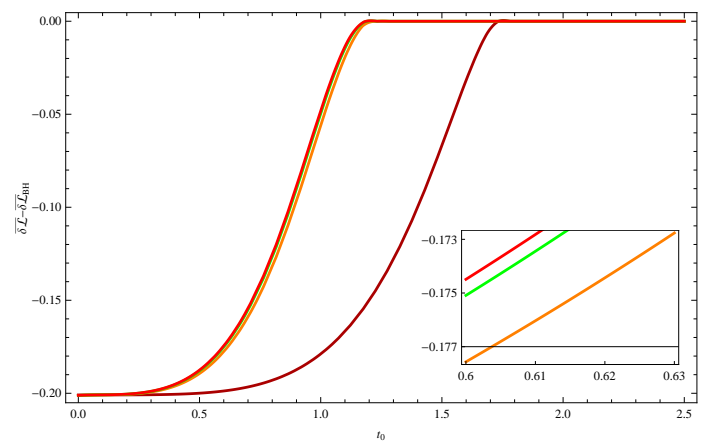

(c) $\mathrm{AdS}_{5}, R_{0}=R_{f}=2$

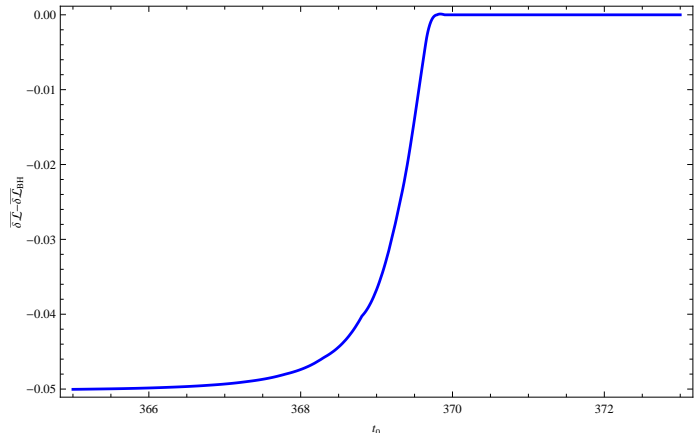

(b) $\mathrm{AdS}_{5}, R_{0}=R_{f}=0.5$

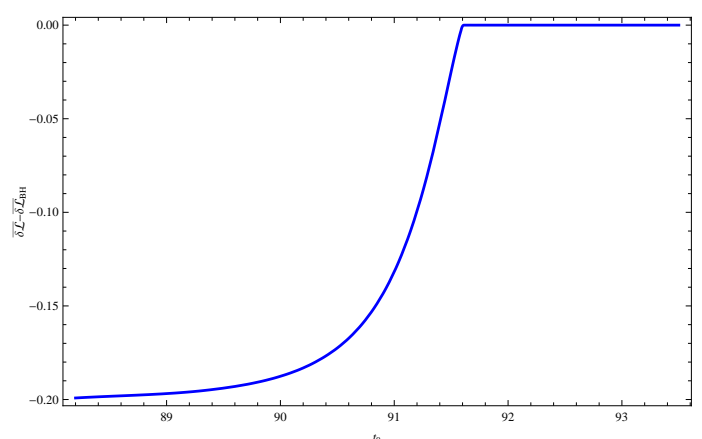

(d) $\mathrm{AdS}_{5}, R_{0}=R_{f}=2$

Figure 2: Renormalized space-like geodesic length as a function of time for Vaidya-type (red curve), scalar field (green curve), massive dust (orange curve), relativistic matter (dark red curve), conformal matter (blue curve) shells, respectively. Insets zoom in the first curve in both figures. 


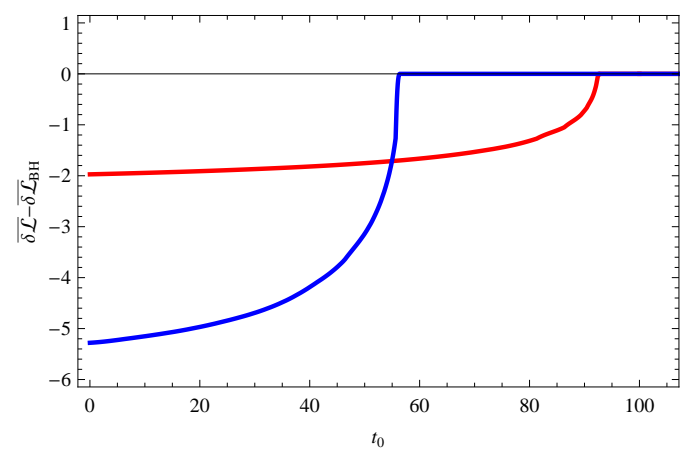

Figure 3: Renormalized geodesic length differences when $R_{0}=0.5 ; R_{f}=1$ (red curve) and $R_{f}=2$ (blue curve).

between both radii. This is so because by changing the radii one varies the velocity of the shell as well as the position $z_{*}$ of the thermalized geodesic tip.

Figure 4, on the other hand, shows a similar behavior as figure 1 for renormalized rectangular minimal area surfaces for $d=3$ and 4 . In this case we also set $R_{0}=R_{f}=1$ and $2 M=1$. We observe the same trend as in figure 1 . The thermalization shown in figure 4 corresponding to rectangular Wilson loops in the dual QFT shows the appearance of swallow tails when thermal equilibrium is reached. Something similar was observed before in the case of an AdS-Vaidya shell [9], and even in the cases with an AdS-Vaidya shell composed by charged dust [17].

\section{Discussion and conclusions}

In this paper we have studied dynamical evolution of thin shells composed by different degrees of freedom in AdS spaces, obtaining different thermalization time scales. We have used the thin-shell formalism, applying the Israel junction conditions, and also imposed the positive energy conditions. Thus, we obtain a general framework where the distinction in the composition of the shells is made explicit through the equation of state in each case. We have also explored different space-time dimensions.

We have considered an AdS-Vaidya shell, which can be understood as composed by massless dust, moving at the speed of light, and then we also investigated shells made of a scalar field, a pressureless massive fluid, the so-called relativistic matter, and matter whose energymomentum tensor is traceless. The parameters to play with are the space-time dimension $d$, and the radii of the inner and outer regions, $R_{0}$ and $R_{f}$.

The first observation is that when the $R_{0}=R_{f}$, the thermalization time scales of the AdS-Vaidya, the scalar field and a pressureless massive fluid shells, are the same. The conformal case thermalizes much later, strongly depending on space-time dimensions and 


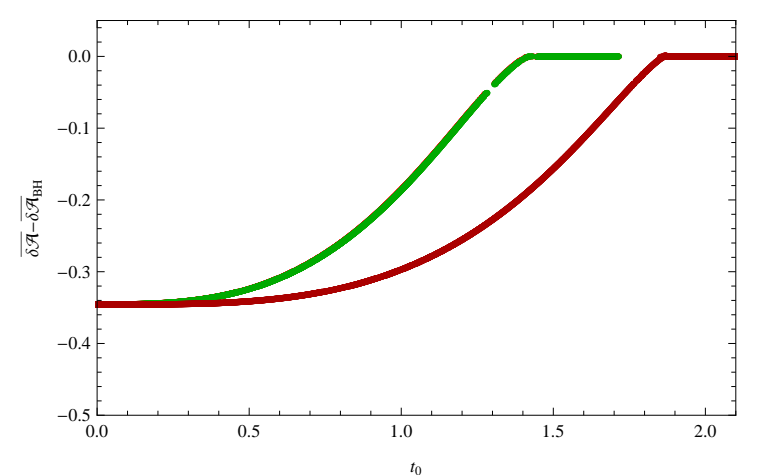

(a) $\mathrm{AdS}_{5}$

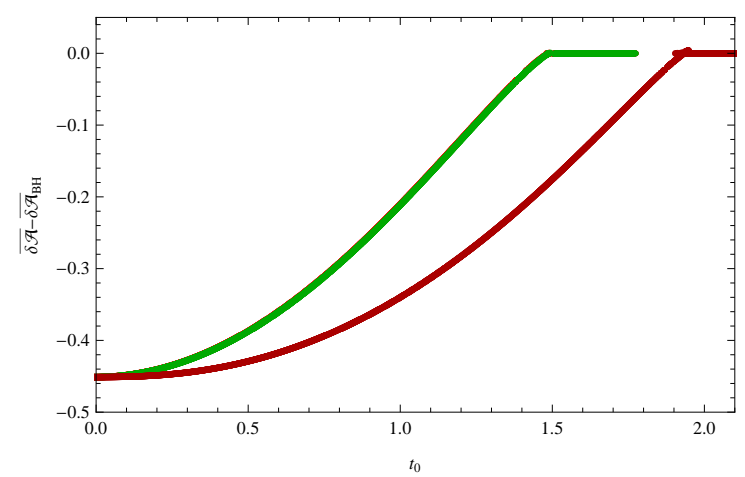

(c) $\mathrm{AdS}_{4}$

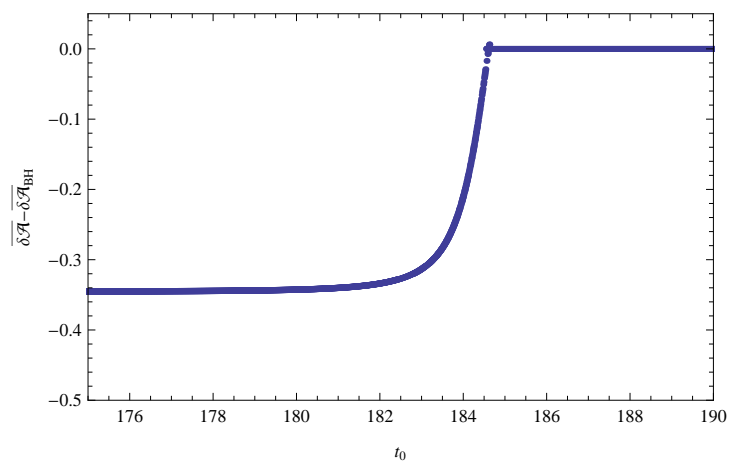

(b) $\mathrm{AdS}_{5}$

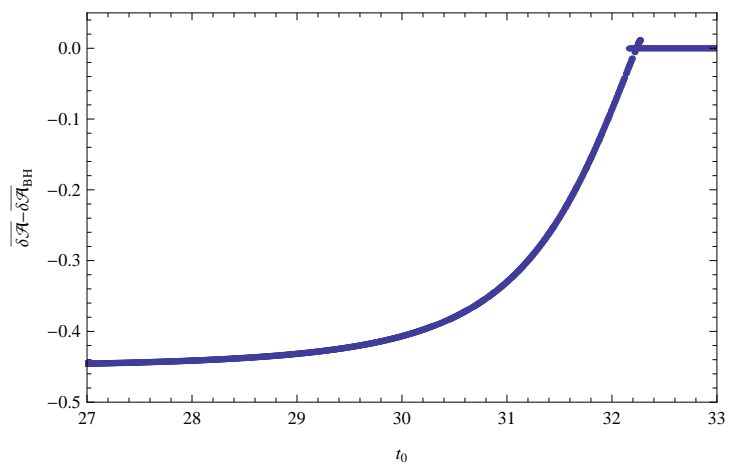

(d) $\mathrm{AdS}_{4}$

Figure 4: Thermalization of the renormalized minimal area surfaces, with $\ell=2$, considering $R_{0}=R_{f}=1$, for the boundary theory dimensions $d=4$ and 3 , labeled by $\mathrm{AdS}_{5}$ and $\mathrm{AdS}_{4}$, respectively. Different kinds of matter in the shell are indicated by colored curves as follows: Vaidya-type (red curve), scalar field (green curve), massive dust (orange curve), relativistic matter (dark red curve), conformal matter (blue curve) shells. 
radii. Relativistic matter case continuously interpolates between both cases, depending on its EOS.

In addition, we have studied the effect on the thermalization curves when the inner and outer radii are different. Also, we have found that the positive energy condition implies that the inner radius must be equal or smaller than the outer one, which means that the absolute value of the vacuum energy density of the inner region must be equal or larger than the one of the outer region. Finally, we have found that only in the case of a shell composed by a scalar field the positive energy condition allows for the shell to collapse.

When the energy densities of the inner and outer spaces differ, the thermalization time scales considerably increases. For instance, for the scalar field case, which for equal radii coincides with the AdS-Vaidya shell, for different radii the thermalization time can be set arbitrarily large. Some particular examples where displayed in figure 3.

The main conclusion from this work is that holographic models do not necessarily yield a rapid thermalization. Moreover, the thermalization time scale strongly depends on the equation of state governing the shell. This will determine the shell velocity and consequently, thermalization times. We show that it is possible to have EOS that lead to delayed thermalization times (such as the case of conformal matter).

There are other possible directions where the ideas and formalism presented here can be extended. For instance, while changing the composition of the shell we will be imposing different shell velocities. This allows one to model different possible scenarios for the evolution of thermalization processes in strongly coupled systems. One is to consider lowerdimensional systems in the context of AdS/CMT. Another aspect concerns the study of a quantum quench across critical points $[49,50]$. For example a quantum quench across a zero temperature holographic superfluid transition has recently been reported in [51]. Another very interesting extension could be along the lines of the recent work by Buchel, Lehner and Myers, where it has been studied thermal quenches in a particular mass deformation of the $\mathcal{N}=4 \mathrm{SYM}$ theory. There is a transition between an initial thermal state of $\mathcal{N}=4 \mathrm{SYM}$, to a final state with the mentioned mass deformation which yields the so-called $\mathcal{N}=2^{*} \mathrm{SYM}$ theory. This transition has been described in terms of a thermal quench [52].

\section{Acknowledgments}

We thank Alex Buchel, Johanna Erdmenger, Nicolás Grandi, Luis Lehner, Shu Lin, Juan Maldacena, Carlos Núñez, Guillermo Silva and Gianmassimo Tasinato for useful discussions and comments. We specially thank Alex Buchel for a critical reading of the manuscript. The work of W.B. and M.S. has been supported by CONICET, the Consejo Nacional de Investigaciones Científicas y Técnicas of Argentina, and the ANPCyT-FONCyT Grant PICT-200700849. The work of M.S. has also been supported by the CONICET Grant PIP-2010-0396. 
Research at Perimeter Institute is supported by the Government of Canada through Industry Canada and by the Province of Ontario through the Ministry of Research \& Innovation. D.G. also acknowledges support from an NSERC Discovery grant. 


\section{References}

[1] J. M. Maldacena, "The large N limit of superconformal field theories and supergravity," Adv. Theor. Math. Phys. 2 (1998) 231 [Int. J. Theor. Phys. 38 (1999) 1113] [arXiv:hepth/9711200].

[2] S. S. Gubser, I. R. Klebanov and A. M. Polyakov, "Gauge theory correlators from non-critical string theory," Phys. Lett. B 428 (1998) 105 [arXiv:hep-th/9802109].

[3] E. Witten, "Anti-de Sitter space and holography," Adv. Theor. Math. Phys. 2 (1998) 253 [arXiv:hep-th/9802150].

[4] S. R. Das, T. Nishioka and T. Takayanagi, "Probe Branes, Time-dependent Couplings and Thermalization in AdS/CFT," JHEP 1007 (2010) 071 [arXiv:1005.3348 [hep-th]].

[5] J. Abajo-Arrastia, J. Aparicio and E. Lopez, "Holographic Evolution of Entanglement Entropy," JHEP 1011 (2010) 149 [arXiv:1006.4090 [hep-th]].

[6] T. Albash and C. V. Johnson, "Evolution of Holographic Entanglement Entropy after Thermal and Electromagnetic Quenches," New J. Phys. 13 (2011) 045017 [arXiv:1008.3027 [hep-th]].

[7] H. Ebrahim and M. Headrick, "Instantaneous Thermalization in Holographic Plasmas," arXiv:1010.5443 [hep-th].

[8] V. Balasubramanian, A. Bernamonti, J. de Boer, N. Copland, B. Craps, E. KeskiVakkuri, B. Muller and A. Schafer et al., "Thermalization of Strongly Coupled Field Theories," Phys. Rev. Lett. 106 (2011) 191601 [arXiv:1012.4753 [hep-th]].

[9] V. Balasubramanian, A. Bernamonti, J. de Boer, N. Copland, B. Craps, E. KeskiVakkuri, B. Muller and A. Schafer et al., "Holographic Thermalization," Phys. Rev. D 84 (2011) 026010 [arXiv:1103.2683 [hep-th]].

[10] D. Garfinkle and L. A. Pando Zayas, "Rapid Thermalization in Field Theory from Gravitational Collapse," Phys. Rev. D 84 (2011) 066006 [arXiv:1106.2339 [hep-th]].

[11] J. Aparicio and E. Lopez, "Evolution of Two-Point Functions from Holography," JHEP 1112 (2011) 082 [arXiv:1109.3571 [hep-th]].

[12] A. Allais and E. Tonni, "Holographic evolution of the mutual information," JHEP 1201 (2012) 102 [arXiv:1110.1607 [hep-th]].

[13] V. Keranen, E. Keski-Vakkuri and L. Thorlacius, "Thermalization and entanglement following a non-relativistic holographic quench," Phys. Rev. D 85 (2012) 026005 [arXiv:1110.5035 [hep-th]]. 
[14] D. Garfinkle, L. A. Pando Zayas and D. Reichmann, "On Field Theory Thermalization from Gravitational Collapse," JHEP 1202 (2012) 119 [arXiv:1110.5823 [hep-th]].

[15] S. R. Das, "Holographic Quantum Quench," J. Phys. Conf. Ser. 343 (2012) 012027 [arXiv:1111.7275 [hep-th]].

[16] V. E. Hubeny, "Extremal surfaces as bulk probes in AdS/CFT," arXiv:1203.1044 [hepth].

[17] D. Galante and M. Schvellinger, "Thermalization with a chemical potential from AdS spaces," JHEP 1207 (2012) 096 [arXiv:1205.1548 [hep-th]].

[18] E. Caceres and A. Kundu, "Holographic Thermalization with Chemical Potential," JHEP 1209 (2012) 055 [arXiv:1205.2354 [hep-th]].

[19] B. Wu, "On holographic thermalization and gravitational collapse of massless scalar fields," JHEP 1210 (2012) 133 [arXiv:1208.1393 [hep-th]].

[20] U. H. Danielsson, E. Keski-Vakkuri and M. Kruczenski, "Black hole formation in AdS and thermalization on the boundary," JHEP 0002 (2000) 039 [hep-th/9912209].

[21] S. B. Giddings and S. F. Ross, "D3-brane shells to black branes on the Coulomb branch," Phys. Rev. D 61 (2000) 024036 [hep-th/9907204].

[22] R. A. Janik and R. B. Peschanski, "Gauge/gravity duality and thermalization of a boost-invariant perfect fluid," Phys. Rev. D 74 (2006) 046007 [hep-th/0606149].

[23] R. A. Janik, "Viscous plasma evolution from gravity using AdS/CFT," Phys. Rev. Lett. 98 (2007) 022302 [hep-th/0610144].

[24] P. M. Chesler and L. G. Yaffe, "Horizon formation and far-from-equilibrium isotropization in supersymmetric Yang-Mills plasma," Phys. Rev. Lett. 102 (2009) 211601 [arXiv:0812.2053 [hep-th]].

[25] P. M. Chesler and L. G. Yaffe, "Boost invariant flow, black hole formation, and farfrom-equilibrium dynamics in $\mathrm{N}=4$ supersymmetric Yang-Mills theory," Phys. Rev. D 82 (2010) 026006 [arXiv:0906.4426 [hep-th]].

[26] S. Bhattacharyya and S. Minwalla, "Weak Field Black Hole Formation in Asymptotically AdS Spacetimes," JHEP 0909 (2009) 034 [arXiv:0904.0464 [hep-th]].

[27] S. Lin and E. Shuryak, "Toward the AdS/CFT Gravity Dual for High Energy Collisions. 3. Gravitationally Collapsing Shell and Quasiequilibrium," Phys. Rev. D 78 (2008) 125018 [arXiv:0808.0910 [hep-th]]. 
[28] E. Shuryak, S. -J. Sin and I. Zahed, "A Gravity dual of RHIC collisions," J. Korean Phys. Soc. 50 (2007) 384 [hep-th/0511199].

[29] W. Fischler and S. Kundu, "Strongly Coupled Gauge Theories: High and Low Temperature Behavior of Non-local Observables," arXiv:1212.2643 [hep-th].

[30] G. Policastro, D. T. Son and A. O. Starinets, "The Shear viscosity of strongly coupled N=4 supersymmetric Yang-Mills plasma," Phys. Rev. Lett. 87 (2001) 081601 [hepth/0104066].

[31] A. Buchel, J. T. Liu and A. O. Starinets, "Coupling constant dependence of the shear viscosity in N=4 supersymmetric Yang-Mills theory," Nucl. Phys. B 707 (2005) 56 [hep-th/0406264].

[32] G. Policastro, D. T. Son and A. O. Starinets, "From AdS / CFT correspondence to hydrodynamics," JHEP 0209 (2002) 043 [hep-th/0205052].

[33] B. Hassanain and M. Schvellinger, "Towards 't Hooft parameter corrections to charge transport in strongly-coupled plasma," JHEP 1010 (2010) 068 [arXiv:1006.5480 [hepth]].

[34] B. Hassanain and M. Schvellinger, "Plasma conductivity at finite coupling," JHEP 1201 (2012) 114 [arXiv:1108.6306 [hep-th]].

[35] S. Caron-Huot, P. Kovtun, G. D. Moore, A. Starinets and L. G. Yaffe, "Photon and dilepton production in supersymmetric Yang-Mills plasma," JHEP 0612 (2006) 015 [hep-th/0607237].

[36] B. Hassanain and M. Schvellinger, "Diagnostics of plasma photoemission at strong coupling," Phys. Rev. D 85 (2012) 086007 [arXiv:1110.0526 [hep-th]].

[37] B. Hassanain and M. Schvellinger, "Plasma photoemission from string theory," arXiv:1209.0427 [hep-th].

[38] R. Baier, S. A. Stricker, O. Taanila and A. Vuorinen, "Holographic Dilepton Production in a Thermalizing Plasma," JHEP 1207 (2012) 094 [arXiv:1205.2998 [hep-ph]].

[39] R. Baier, S. A. Stricker, O. Taanila and A. Vuorinen, "Production of Prompt Photons: Holographic Duality and Thermalization," Phys. Rev. D 86 (2012) 081901 [arXiv:1207.1116 [hep-ph]].

[40] D. Steineder, S. A. Stricker and A. Vuorinen, "Thermalization at intermediate coupling," arXiv:1209.0291 [hep-ph]. 
[41] W. Israel, "Singular hypersurfaces and thin shells in general relativity," Nuovo Cim. B 44S10 (1966) 1 [Erratum-ibid. B 48 (1967) 463] [Nuovo Cim. B 44 (1966) 1].

[42] P. C. Vaidya, "The external field of a radiating star in general relativity", Curr. Sci. 12 (1943) 183.

[43] P. C. Vaidya, "The gravitational field of a radiating star", Proc. Indian. Acad. Sci. A. 33 (1951) 264

[44] J. Erdmenger and S. Lin, "Thermalization from gauge/gravity duality: Evolution of singularities in unequal time correlators," JHEP 1210 (2012) 028 [arXiv:1205.6873 [hepth]].

[45] S. Blau, E. Guendelman, A. Guth, "Dynamics of false-vacuum bubbles", Phys.Rev.D 35 (1987) 35.

[46] V. Balasubramanian and S. F. Ross, "Holographic particle detection," Phys. Rev. D 61 (2000) 044007 [hep-th/9906226].

[47] J. M. Maldacena, "Wilson loops in large N field theories," Phys. Rev. Lett. 80 (1998) 4859 [hep-th/9803002].

[48] S. -J. Rey and J. -T. Yee, "Macroscopic strings as heavy quarks in large N gauge theory and anti-de Sitter supergravity," Eur. Phys. J. C 22 (2001) 379 [hep-th/9803001].

[49] P. Calabrese and J. L. Cardy, "Time-dependence of correlation functions following a quantum quench," Phys. Rev. Lett. 96 (2006) 136801 [cond-mat/0601225].

[50] P. Calabrese and J. Cardy, "Quantum Quenches in Extended Systems," J. Stat. Mech. 0706 (2007) P06008 [arXiv:0704.1880 [cond-mat.stat-mech]].

[51] P. Basu, D. Das, S. R. Das and T. Nishioka, "Quantum Quench Across a Zero Temperature Holographic Superfluid Transition," arXiv:1211.7076 [hep-th].

[52] A. Buchel, L. Lehner and R. C. Myers, JHEP 1208 (2012) 049 [arXiv:1206.6785 [hepth]]. 